\title{
Erdöl, ökonomische Krise und der Staat in Mexiko
}

\author{
von Herbert Baumgartner
}

Das boomartige Wachstum der mexikanischen Okonomie zwischen 1978 und 1981, sowie die folgende jähe Rezession, die allenfalls mit den Auswirkungen der Krise der 1930er Jahre verglichen werden kann, läßt zwangsläufig die Frage nach den Ursachen dieser Entwicklung aufkommen. Die Schärfe der Kontraktion ist umso erstaunlicher, wenn die relativ günstigen Ausgangsbedingungen Mexikos in Betracht gezogen werden: Die Revolution von 1910-1917 schuf eine Ideologie gesellschaftlicher Gleichheit, die später unter anderem in einer weitreichenden Agrarreform umgesetzt wurde. Trotz aller Abhängigkeit von den USA waren die Voraussetzungen nationaler Selbstbestimmung früh gegeben. Seit etwa 1940 bildete sich durch die Nationalisierung von Schlüsselindustrien und eine Wirtschaftspolitik der Importsubstitution eine relativ diversifizierte und entwickelte Industriestruktur mit einem potentiell großen Binnenmarkt heraus. Der Finanzsektor liegt in nationaler Hand. Durch umfangreiche Investitionen, vor allem in die Infrastruktur, sorgte der Staat für anhaltend hohes Wachstum. Seit der Wiederentdeckung des, durch gestiegene Preise rentablen, mexikanischen Erdöls ab 1974, ergab sich durch die neuerlangte Kreditwürdigkeit eine hohe Verschuldungsmöglichkeit bei ausländischen Geldgebern, die durch steigende Einnahmen aus den Ollexporten zunehmend ergänzt wurde.

Neben den Auswirkungen der weltweiten Krisensituation lassen sich vor allem zwei, näher zu untersuchende, interne Ursachen für den ökonomischen Sturzflug nennen. Einerseits ist ein "Ölrauschsyndrom", gekennzeichnet durch weitreichende Korruption und ineffiziente Verwendung finanzieller Ressourcen, für den kräftigen negativen Ausschlag des ökonomischen Barometers verantwortlich. Andererseits lassen die gesellschaftlichen und wirtschaftlichen Grundstrukturen Mexikos auch langfristig eine Umsetzung von Wachstum in eine Entwicklung im Sinne akkumulationsfähiger Gesellschaftsstrukturen und der Befriedigung von Grundbedürfnissen zweifelhaft erscheinen.

\section{Der Ölsektor und die PEMEX}

Die beeindruckendsten Erfolge in der ökonomischen Entwicklung der letzten Jahre wurden sicherlich im Olsektor erzielt. Die Tabelle zeigt den sprunghaften Anstieg sowohl der nachgewiesenen Erdölreserven, der Olproduktion als auch der Olexporte. Mexiko wurde nach der UdSSR, den USA und Saudi Arabien zum viertgrößten 
Ölproduzenten der Welt. ${ }^{1}$ Obwohl die Explorationstätigkeit der staatlichen Ölfirma Petróles Mexicanos - Pemex - seit 1968 kontinuierlich abnahm, ${ }^{2}$ wurden erst mit dem Präsidentenwechsel 1976 wesentlich höhere Reserven bekanntgegeben. Dies bildet einen Hinweis darauf, daß der Start in den Olboom neben den gestiegenen Weltmarktpreisen vor allem eine politische Entscheidung war.

Seit der Erschließung der Reforma Ölfelder in den südlichen Bundesstaaten Tabasco und Chiapas 1972 und der Campeche off-shore Felder in der angrenzenden südlichen Golfregion 1976 werden in diesen beiden Gebieten $90 \%$ des gesamten Erdöls produziert. 1982 wurden 2,7 mio Barrel pro Tag (b/d) gefördert. ${ }^{3}$ Die anfängliche Strategie einer jährlichen Förderung bis zu maximal 1/20 der nachgewiesenen Reserven, wurde durch die Bekanntgabe immer größerer Vorkommen spätestens 1978 hinfällig. Eine "Wachstumsstrategie um jeden Preis" kann daran ermessen werden, daß sowohl die PemexPlanung von 1975 bis 1977 wesentlich früher und mit einer höheren Produktion erreicht wurde, und daß viele Voraussagen übertroffen wurden. ${ }^{4}$ Erst das Energieprogramm von 1980 sah eine Produktionsplattform von maximal 2,7 mio b/d vor. ${ }^{5}$ Dem gingen heftige innenpolitische Diskussionen über die Verwendung der Olressourcen voraus. Die linke Opposition setzte sich für eine behutsame Förderpolitik ein, um die Absorptionsfähigkeit der Ókonomie für die Ollfelder sicherzustellen und die ausländische Ausbeutung zu verringern. Die Festsetzung einer Höchstfördermenge war aber nur oberflächlich betrachtet ein politisches Zugeständnis. Tatsächlich überragten jedoch die technischen Schwierigkeiten. Die Hafenkapazitäten waren zu klein für eine höhere Verschiffungsquote, die Lagerkapazitäten reichten nicht aus, und es mußte zudem ein sehr hoher Anteil des assoziierten Erdgases abgefackelt werden. ${ }^{6}$

Aufgrund des weltweiten Erdölüberangebots ab 1981 entschied sich auch die neue

1 Pemex, Anuario Estadistico, México 1983, S. 17. Da erst ca. $15 \%$ des mexikanischen Territoriums exploriert sind, belaufen sich die wahrscheinlichen Reserven auf weitere $90 \mathrm{mrd}$ Barrel, die potentiellen auf $250 \mathrm{mrd}$ Barrel. Zum Vergleich: die nachgewiesenen Reserven Saudi Arabiens betragen $150 \mathrm{mrd}$. Barrel. Vgl. Uno más uno, 19. 3. 1983, suplemento, S. 8.

21968 wurden 602 Bohrlöcher erschlossen mit einer Gesamttiefe von $1447 \mathrm{~km}$. Dieser Anteil sank auf 431/ 1136 k m 1972 und 333/975 k m 1979. Secretaría de Programación y Presupuesto/Pemex, La Industria Petrolera en México, México 1980, S. 85.

3 Vgl. Beteta, M. R., Informe del director general de Pemex, in: Uno más uno, suplemento, 19. 3. 1983, S. 4; Grossling, B. F., Possible Dimensions of Mexican Petroleum, in: Ladman, J. R., et al., U.S. - Mexican Energy Relationships, Lexington, Mass. 1981, S. 39; Mancke, R. B., Mexican Oil and Natural Gas, New York 1979, S. 63.

4 Zum Wachstum um jeden Preis: Carmona, F., México 1979: el "salvavidas" del petróleo y la estrategía del régimen, in: Estrategía, (25), 1979, S. 13: Williams, E. J., The Rebirth of the Mexican Petroleum Industry, Lexington, Mass. 1979, S. 102: Villar, S. I., Estado y petróleo en México: experiencias y perspectivas, in: Foro Internacional, (20-1), 1978, S. 139. Für die höhere Planerfüllung: Yunez Naude, A., Política petrolera y perspectivas de desarrollo de la economía mexicana, in: Las perspectivas del petróleo mexicano, El Colegio de México, México 1979, S. 209; Plan Basico de Gobierno 1976-1982, Partido Revolucionario Institucional, México 1975, S. 113, der noch die sehr bescheidene Produktion von 1,2 mio b/d für 1982 annimmt.

5 Programa de Energía, in: El Mercado de Valores, (40-47), 1980, S. 1173.

6 Vgl. Uno más uno, 11. 4. 1983, S. 7. Bei der Offshore-Förderung stieg die Ausnutzung des Gases in der Zwischenzeit von fast $0 \% 1980$ auf $42 \%$ 1982. Beteta, (Fn. 3), S. 4. 
Regierung 1983 das Förderlimit beizubehalten, wobei ursprüngliche Pläne für längerfristig wesentlich höhere Exporte zurückgestellt wurden. ${ }^{7}$ Das Energieprogramm von 1980 sieht auch eine, noch gültige, Begrenzung der Exporte auf 1,65 mio b/d und 300 mio Kubikfuß Gas pro Tag (cf/d) vor. Die Bestimmung, daß kein Land mehr als $50 \%$ der mexikanischen Ölexporte erhalten kann, war gegen den hohen Exportanteil der USA gerichtet. Von 1979 bis 1982 wurde dieser Anteil deshalb von $84 \%$ auf $49 \%$ abgesenkt. Real stieg der US-Import mexikanischen Öls aber von $0,448 \mathrm{mio} \mathrm{b} / \mathrm{d}$ auf 0,548 mio b/d. Dadurch wurde Mexiko 1982 zum größten Ölimporteur in die USA.$^{8}$ Die Gasleitung in die USA, deren Bau bis 1979 stark umstritten war, hat in der Folgezeit nur eine bescheidene Bedeutung erlangt. Die kontraktierte Höchstmenge von $300 \mathrm{mio} \mathrm{cf} / \mathrm{d}$ wurde bis 1983 nicht erreicht. ${ }^{9}$

1982 belieferte Mexiko 25 Länder, wobei Spanien, Japan und Frankreich die nächstwichtigeren Kunden sind. Die Diversifizierung der Exporte in Bezug auf die europäischen und japanischen Kunden soll verstärkt Direktinvestitionen und damit einen höheren Technologietransfer bringen. Um den politischen Einfluß der USA abzuschwächen, liefern seit 1980 Venezuela und Mexiko gemeinsam an 10 karibische und mittelamerikanische Länder zu Sonderbedingungen bis zu 0,32 mio b/d. ${ }^{10}$

Seit März 1983 änderte Mexiko seine bis dahin auf Unabhängigkeit bedachte Politik gegenüber der OPEC. Mexiko abzeptierte die Exportquote von 1,65 mio b/d und richtet seine Exportpreise nach denen der OPEC. Mexiko wird damit zu einem Zeitpunkt praktisch zu einem Mitglied der OPEC, zu dem eine weitere Expansion der Exporte nicht mehr möglich ist. Zuvor konnten im Schatten des OPEC-Kartells die Exporte 1981 und 1982 noch trotz des weltweiten Rohölüberangebots um $55 \%$ erhöht werden. Trotz der Expansion der Exporte mußten wegen des Verfalls des Olpreises auf den internationalen Märkten die Einnahmenerwartungen von etwa 20 mrd US \$ pro Jahr auf $15 \mathrm{mrd}$ US \$ reduziert werden. ${ }^{11}$ Die Petrolisierung der mexikanischen Exporte nahm erheblich zu: 1977 betrug der Anteil des Erdöls an den Gesamtexporten 24 \%, $198062 \%$ und 1982 $74 \% .^{12}$

Bereits von 1917 bis 1921 förderte Mexiko $25 \%$ der Weltölproduktion. Die Verlagerungsmanöver der britischen und amerikanischen Olkonzerne hin zur kostengünstigeren

7 Beteta, (Fn. 3), S. 5: Begrenzung der Förderung von Rohöl auf 2,75 mio b/d und 4300 cf/d Gas. Für Mitte der 80er Jahre wurde bereits mit einer Förderung von 4 mio b/d gerechnet. Vgl. Thumser, G., Reichtum schafft Armut, Mexiko: Der Olgigant ist pleite, in: Links, (14-153), 1982, S. 13; Puente Leyva, J., Mexico: Petroleum Perspectives, in: Ladman, (Fn. 3), S. 19.

8 Vgl. Beteta, (Fn. 3), S. 4; SPP/Pemex, (Fn. 2), S. 285; Excelsior, 16. 8. 1983, S. A-1; Uno más uno, 15. 3. 1983, S. 6.

91982 wurden insgesamt nur $90 \%$ der Menge an Gas exportiert $=478$ mio US \$.

101983 wurde der Pacto de San José allerdings zu etwas schlechteren Bedingungen für die Importeure verlängert. Excelsior, 30. 6. 1983, S. F-2.

11 Seit Mai 1981 sank der Preis für die Istmo-Qualität von 38,5 auf 29 US \$/b im März 1983. Vgl. Beteta, (Fn. 3), S. 4; Uno más uno, 15. 3. 1983, S. 1.

12 Berechnet nach Presidencia de la República, Primer Informe de Gobierno 1983, Sector Política Económica, México 1983 und Banco de México, Informe Anual 1982, México 1983. 
Produktion in Venezuela senkten die mexikanische Olproduktion bis 1938 um $80 \% .^{13}$ Durch die Verstaatlichung der Olindustrie 1938, zu der sich die populistische Regierung Cárdenas' durch zunehmende politische Schwierigkeiten gezwungen sah, war die Pemex frühzeitig darauf angewiesen, eigene Technologien zu entwickeln. Mit dem Aufbau eines eigenen Forschungsinstituts, das 1982 etwa 3500 Mitarbeiter zählte, konnte die technologische Basis weiter verbessert werden, so daß die Pemex technologisches know how bereits in andere Länder der Dritten Welt exportiert. ${ }^{14}$ Da die Pemex von der Exploration bis zur Distribution sämtliche Verarbeitungsprozesse selbst kontrolliert, spricht der Pemex-Direktor wohl zurecht von der vertikal integriertesten Ölgesellschaft der Welt. Außerdem besteht sowohl in der Olraffinierung als auch in der Weiterverarbeitung der Basispetrochemie eine breite Diversifizierung. Der Raffineriesektor deckt vor allem den internen Verbrauch. Die Verarbeitungskapazität soll von derzeit 1,6 mio b/d auf 1,8 mio b/d 1988 erhöht werden. ${ }^{15}$

Die Petrochemie sollte zum zentralen Bestandteil einer Exportdiversifizierung werden, bei der das Rohöl unter dem Monopol der Pemex in Mexiko selbst weiterverarbeitet wird. Von 1977 bis 1982 hat sich die petrochemische Produktion verdoppelt und sollte 1990 bei 23 mio t pro Jahr liegen. Durch die ökonomische Krise mußten die Pläne allerdings reduziert werden. Wenn auch der interne Bedarf beinahe gedeckt werden kann und ab 1984 mit einem relativ bescheidenen Exportüberschuß von 0,2 mrd US $\$$ bei den petrochemischen Produkten gerechnet wird, scheint ein hoher Exportanteil der Petrochemie auf absehbare Zeit nicht verwirklichbar. ${ }^{16}$

Die ökonomische Bedeutung der Pemex als größtes lateinamerikanisches Unternehmen ist für Mexiko erheblich. Der Beitrag der Pemex zum Inlandsprodukt hat den der Landwirtschaft überholt. Bedeutender ist aber der Anteil an den öffentlichen Investitionen und am Steueraufkommen. 1982 betrugen die Steuerzahlungen der Pemex 11,8 mrd US \$, die Investitionen 5,6 mrd US \$, wobei die Pemex $12 \%$ des gesamten mexikanischen Anlagekapitals besitzt. ${ }^{17}$ Die Pemex gibt ihre Olprodukte im Inland weit unter den Weltmarktpreisen ab und leistet damit erhebliche Transfers an die Verbraucher. Obwohl

13 Vgl. Furtado, C., Economic Development of Latin America, Cambridge 1970, S. 189; Mancke, R. B., Mexican Oil and Natural Gas. New York 1979, S. 47.

14 SPP/Pemex, (Fn. 2), S. 359. Interviewergebnisse. Auf der höchsten technologischen Ebene der Olindustrie, insbesondere in der Petrochemie, beträgt die ausländische Abhängigkeit noch etwa $30 \% .1982$ konnte die Pemex die erste offshore Plattform komplett selbst herstellen.

15 Beteta, (Fn. 3), S. 4; García-Colín Scherer, L., La ciencia y la tecnología del petróleo, Situación actual y perspectivas futuras en México, in: Foro Internacional, (18-4), 1978, S. 689. Vgl. auch Proceso, (333), 21. 3. 1983, S. 15; Excelsior, 18. 7. 1983, S. A-4.

16 Vgl. Ehrke, M., Internationalisierte Produktion in Mexiko, in: Vierteljahresberichte, Probleme der Entwicklungsländer, (87), 1982, S. 63; La industria petroquimica en México, Centro de Información y Estudios Nacionales, México 1982, S. 2-6; Uno más uno, 19. 3. 1983, S. 8 und 13. 4. 1983, S. 8; Financial Times, 12. 8. 1981.

17 Siehe dazu vor allem die Tabelle. Vgl. auch Nohlen, D.,/Lehr, V. G., Mexiko, in: Handbuch der Dritten Welt, Bd. 3, Mittelamerika und Karibik, Hamburg 1982, S. 132; Beteta, (Fn. 3), S. 4; Pemex, Anuario Estadistico 1982, México 1983, S. 144. 
Aktivitäten der Pemex

\begin{tabular}{|c|c|c|c|c|c|c|}
\hline & Einheit & 1973 & 1974 & 1975 & 1976 & 1977 \\
\hline $\begin{array}{l}\text { Nachgewiesene Reserven } \\
\text { (Erdôl und Erdgas }\end{array}$ & $\begin{array}{l}\text { mrd } \\
\text { Barrel }\end{array}$ & 5,4 & 5,7 & 6,3 & 11,2 & 16,0 \\
\hline Dauer der Reserven & Jahre & 16 & 14 & 14 & 22 & 29 \\
\hline $\begin{array}{l}\text { Produktion } \\
\text { (Erdöl und Erdgas) }\end{array}$ & $\begin{array}{l}\text { mio } \\
\text { Barrel }\end{array}$ & 335 & 402 & 464 & 500 & 545 \\
\hline davon: Rohölexport & $\begin{array}{l}\text { mio } \\
\text { Barrel }\end{array}$ & 0 & 6 & 34 & 34 & 73 \\
\hline Raffinerieproduktion & $\begin{array}{c}\text { mio } \\
\text { Barrel }\end{array}$ & 206 & 234 & 240 & 268 & 300 \\
\hline $\begin{array}{l}\text { Petrochemische } \\
\text { Produktion }\end{array}$ & mio $t$ & 2,6 & 2,9 & 3,6 & 3,9 & 4,2 \\
\hline$\frac{\text { Pemex - Finanzen: }}{\text { Gesamtumsatz }}$ & mrd US\$ & 1,5 & 2,6 & 3,1 & 2,9 & 3,4 \\
\hline davon: Export & mrd US\$ & 0,0 & 0,1 & 0,5 & 0,5 & 1,0 \\
\hline $\begin{array}{l}\text { Auslands- } \\
\text { verschuldung }\end{array}$ & mrd US\$ & 0,7 & 0,9 & 1,7 & 2,2 & 3,0 \\
\hline$\frac{\text { Pemex-Anteil }}{\text { am Inlandsprodukt }}$ (BIP) & $\%$ & 2,5 & 3,1 & 3,2 & 3,0 & 3,8 \\
\hline $\begin{array}{l}\text { an den öffentlichen } \\
\text { Investitionen }\end{array}$ & $\%$ & 15,5 & 15,7 & 15,3 & 19,5 & 23,6 \\
\hline am steueraufkommen & $\%$ & 3,3 & 5,4 & 6,9 & 5,9 & 8,6 \\
\hline Beschäftigte & $\begin{array}{l}\text { tausend } \\
\text { Personen }\end{array}$ & 76,6 & 77,6 & 81,2 & 88,0 & 91,7 \\
\hline
\end{tabular}

gued.len:5 Pemex:72Anuarionstadistico41882. Instituto Mexicano del 
sich die Benzinpreise von 1980 bis 1983 etwa verzehnfacht haben, liegen sie immer noch erst bei $1 / 3$ der europäischen. ${ }^{18}$

Mit dem Regierungswechsel im Dezember 1982 wurde auch die gesamte Führungsmannschaft der Pemex ausgewechselt. Da der neue Generaldirektor Beteta aus der staatlichen Somex Bank kam, wurden auch die restlichen Direktorenposten mit Bankern besetzt. Seither wird die frühere Pemex, insbesondere unter der Leitung Diaz Serranos bis zum Sommer 1981, von der neuen Direktion selbst als ineffizient und korrupt bezeichnet: Die sehr schnelle Expansion der Pemex seit 1976 verstärkte die bereits vorher ineffizienten Strukturen und führte zu einer hohen Unabhängigkeit von staatlicher Aufsicht; das zu schnelle Wachstum hat zu Ungleichgewichten innerhalb der Pemex geführt; es fehlte eine integrale Planung; Gigantismus und eine aufgeblähte Bürokratie waren die Folge. In anderen Studien wird von administrativem Chaos und unerklärlicher Autonomie der höheren Leitungsebenen gesprochen. ${ }^{19} \mathrm{Im}$ Vergleich zu anderen Ölgesellschaften besitzt die Pemex eine sehr niedrige Arbeitsproduktivität, da eine erhebliche personelle Úbersetzung besteht, für die die traditionell starke Olarbeitergewerkschaft verantwortlich ist. ${ }^{20}$

Ebenfalls seit dem Regierungswechsel und im Zuge einer Kampagne zur "moralischen Renovation" wurden viele Korruptionsfälle der vorigen Pemex-Administration aufgedeckt. Prominentestes Opfer war der frühere Generaldirektor Diaz Serrano selbst, der noch 1981 als einer der möglichen Präsidentschaftskandidaten genannt wurde. Beim Kauf von zwei Gastankern hatte er sich 1980 eine Kommission von 34 mio US \$ eingeräumt. In 16 weiteren Fällen summieren sich Bestechung und Korruption im Zusammenhang mit der Pemex auf noch einmal 150 mio US $\$ .{ }^{21}$ Bereits 1979 stellte der Führer der Arbeiterpartei PMT Heberto Castillo das Fehlen von 317 mio Barrel Rohöl mit einem Marktwert von 10 mrd US \$ beim Vergleich verschiedener Statistiken fest, wobei auch bei den Pemex-Einnahmen eine Differenz von 5 mrd US \$ auftrat. Obwohl die Pemex behauptet nie auf Spotmärkten zu verkaufen, tauchten nach Angaben der OPEC 1981 doch erhebliche Mengen mexikanischen Ols auf dem Rotterdamer Markt auf. Für 1980 gaben die USA ihre Olkäufe in Mexiko mit 12 mrd US $\$$ an, die Pemex ihre Verkäufe in die USA aber nur mit $8,84 \mathrm{mrd}$ US $\$ .^{22}$

18 Pemex, Anuario Estadistico 1982, México 1983, S. 129. Die klare Aussage, daß der Transfercharakter billiger Olprodukte durch steigende Preise auch weiterhin abgebaut wird: de la Madrid Hurtado, M., Los grandes problemas nacionales de hoy, México 1982, S. 310.

19 Plan Nacional de Desarrollo 1983-1988, Poder Ejecutivo Federal, México 1983, S. 326; Beteta, (Fn. 3), S. 6-7; Proceso, (348), 4. 7. 1983, S. 9 und (346), 20. 6. 1983, S. 6; Uno más uno, 19. 3. 1983, S. 1 und 13. 4. 1983, S. 8. Für die Entwicklung der ineffizienten Strukturen ab 1938 siehe Sepúlveda, I., PEMEX in a Dependent Society, in: Ladman (Fn. 3), S. 50.

20 Megateli, A., Investment Policies of National Oil Companies, A Comparative Study of Sonatrach, Nioc and Pemex, New York 1980, S. 188; Mancke, (Fn. 13), S. 79; Sachse, M., Ein Riese der vor Kraft nicht laufen kann, in: Finanz und Wirtschaft, 29. 7. 1978.

21 Zusammengestellt aus mexikanischen Tages- und Wochenzeitungen zwischen März und September 1983. Hauptsächlich Excelsior, 30.6. 1983, S. A-1 und 26. 7. 1983, S. A-5.

22 Excelsior, 18. 3. 1983, S. A-22; 30. 6. 1983, S. A-3; Proceso, (348), 4. 7. 1983, S. 13-15; (350), 18. 7. 1983, S. 6. Selbst Pemex-intern sind für die Zeit von 1977 bis Anfang 1980 nur widersprüchliche Produktionszahlen erhältlich. Interviewmaterial. 
Ein öffentlicher Streit der Führer der Ölarbeitergewerkschaft STPRM brachte ebenfalls konkretere Kenntnisse über deren korrupte Strukturen. Die gegenseitigen Korruptionsbeschuldigungen belaufen sich auf etwa 160 mio US $\$ .^{23} \mathrm{Da}$ derart hohe Summen von Gewerkschaftern überhaupt angeeignet werden können, ist lediglich durch die enge Verflechtung der STPRM mit den Pemex-Geschäften zu erklären: Aus allen Investitionstätigkeiten der Pemex erhält die STPRM $2 \%$ für soziale Aufwendungen zugunsten der Arbeiter; die STPRM hat das Recht, $50 \%$ aller Pemex-Bauaufträge einschließlich der Olbohrungen selbst zu kontraktieren; die STPRM übernimmt sämtliche Landtransporte der Pemex von Material und Ausrüstung. Darüberhinaus hat die STPRM seit 1936 das Recht, bis auf etwa $10 \%$ des "Vertrauenspersonals", sämtliche Arbeitsverträge der Pemex selbst abzuschließen. ${ }^{24}$ Seit 1942 haben die Pemex-Arbeiter darüberhinaus das Recht, ihre Arbeitsplätze an nächste Verwandte weiterzugeben, weshalb etwa $50 \%$ der eingestellten Arbeiter mit anderen verwandt sind. ${ }^{25}$

Es bleibt zu bezweifeln, ob in der Konsolidierungsphase seit 1983 die Korruption und die Ineffizienz der Pemex verändert werden kann, obwohl einige Ansätze zu einer administrativen Verbesserung auf höchster Ebene gemacht wurden. ${ }^{26}$ Trotz ihrer Mängel stieg die Bedeutung der Pemex in der mexikanischen Okonomie erheblich an und sie konnte zumindest teilweise ihrer Rolle zur Schaffung zusätzlicher Finanzierungsmittel für den Staat gerecht werden. Dies ist hauptsächlich auf das hohe Rentenmoment der Olförderung zurückzuführen. Im folgenden bleibt zu untersuchen, weshalb über den Ölsektor jedoch keine positiven Auswirkungen auf die Gesamtentwicklung erreicht werden konnte.

\section{Die Krise ab 1982}

Von 1978 bis 1981 wurde der Olsektor seiner Antriebsfunktion für die mexikanische Okonomie durchaus gerecht. Der Ölrausch hatte nicht nur die Regierung, die einheimischen Unternehmer und die Gewerkschaften erfaßt, sondern auch die internationale Bankenwelt, die Mexiko den Anstieg der Auslandsschuld von 23,4 mrd US \$ 1976 auf 81 mrd 1982 finanzierte. Die Krise von 1976 konnte schnell überwunden werden. Verbunden mit einem Anstieg der Investitionsquote am Bruttoinlandsprodukt (BIP) von 19,6\%

23 Excelsior, 12. 9. 1983, S. A-22; Proceso, (354), 15. 8. 1983, S. 10-12; (356), 29. 8. 1983.

24 S. 6. Für Lebenszeitverträge müssen an die STPRM-Führer bis zu 4000 US \$ bezahlt werden, wobei allerdings die Löhne 4 mal höher als im Durchschnitt liegen.Proceso, (350), 18. 7. 1983, S. 12; (353), 8. 8. 1983, S. 14-17; Prevot-Schapira, M.-F., Trabajadores del petróleo y poder sindical en México, in: Wionczek, M. S., Energía en México, México 1982, S. 152.

25 Sepúlveda, (Fn. 19), S. 48; Prevot-Schapira (Fn. 24), S. 147; Randall, L., The Political Economy of Mexican Oil 1976-1979, in: Ladman, (Fn. 3), S. 98; Ehrke, M., Wirtschaftspolitik und staatlicher Sektor in Mexiko, Diss., Universität Hannover 1980, S. 212.

26 Beteta, (Fn. 3), S. 5; Uno más Uno, 13. 4. 1982, S. 1. Auf die Beschreibung der negativen Auswirkungen der Pemex-Tätigkeit in den einzelnen Olprovinzen muß hier verzichtet werden. Siehe dazu vor allem: Allub, L./ Michel, M. A., Impactos regionales de la política petrolera en México, México 1982. 
1977 auf 25,7\% 1981, konnte zwischen 1978 und 1981 ein durchschnittliches Wachstum von $8,4 \%$ erreicht werden. Die Anzahl der Arbeitsplätze konnte um durchschnittlich 5,4\% angehoben werden. ${ }^{27}$ Damit wurden selbst die Erwartungen des Entwicklungsplans für 1980-1982 übertroffen. ${ }^{28}$

Der Preis, der für diese Expansion bezahlt werden mußte, war allerdings sehr hoch. Die öffentliche Verschuldung am BIP stieg von 5,2 \% 1977 auf 17,7 \% 1982. Zwischen 1978 und 1981 addierte sich der negative Saldo der Handelsbilanz trotz der Olexporte auf 12,7 mrd US \$. ${ }^{29}$ Der Vertrauensschwund in die mexikanische Ókonomie manifestierte sich bereits in der "kleinen" Krise des Sommers 1981, als wegen des weltweiten Erdölüberangebots der Olexportpreis um 4 US \$ pro Barrel zurückgenommen wurde. Die Aktienkurse fielen in sechs Monaten um $30 \%$; innerhalb weniger Wochen wurden 5 mrd US \$ in die USA transferiert. Das endgültige Scheitern der Wachstumsstrategie über das Erdöl, verbunden mit ausländischer Verschuldung und einem massiven deficit spending des Staates, wurde im August 1982 mit der Erklärung der externen Zahlungsunfähigkeit klar. 1982 verringerte sich das BIP zum ersten Mal seit 50 Jahren, die Inflation stieg auf beinahe $100 \%$, die of ene Arbeitslosigkeit verdoppelte sich und der mexikanische Peso wurde in einem Jahr um $570 \%$ von 26 auf 148 pro US $\$$ abgewertet. $^{30}$

Als Gegenleistung für eine internationale Rettungsaktion der Banken, in Form einer Umschuldung auf langfristige Kredite und der Stundung des Schuldendienstes, mußte ein Sanierungsprogramm des Internationalen Währungsfonds akzeptiert werden. Mit der Ankündigung eines 10-Punkte Programmes führte die neue Regierung ab Dezember 1982 dieses austeritäre Projekt konsequent durch. Im Mittelpunkt steht dabei eine Verringerung des Haushaltsdefizits am BIP von 17,7\% auf 8,5\% 1983 und 5,5\% 1984, die durch Ausgabenkürzungen und erhöhte Steuereinnahmen erreicht werden soll. Zusammen mit einem verringerten Wachstum der Geldumlaufmenge soll damit die Inflation bekämpft werden. Durch eine drastische Reduzierung der Importe bei gleichzeitiger Förderung der Exporte, vor allem der verarbeitenden Industrie, soll die internationale Zahlungsfähigkeit Mexikos als weiterer Schwerpunkt wieder hergestellt werden. ${ }^{31}$

Seit August 1983 erhält die mexikanische Regierung als Musterbeispiel einer erfolgreichen ökonomischen Konsolidierung den Beifall sowohl der Weltbank, des IWF als auch der internationalen Gläubiger insgesamt: Die Inflationsrate kann 1983 wahrscheinlich auf $80 \%$ abgesenkt werden; nach einem Handelsbilanzdefizit von 4,5 mrd US \$ 1981

27 Berechnet nach Presidencia, (Fn. 12), S. 369, 450, 577; Banco de México, (Fn. 12), S. 72.

28 Plan Global de Desarrollo 1980-1982, Secretaría de Programación y Presupuesto, México 1980, S. 162; Centro de Investigación y Docencia Económicas, Economia Mexicana, Análisis y Perspectivas, Núm. 4, México 1982, S. 10: Für 1981 war das BIP Wachstum auf $8 \%$ geplant, die Arbeitsplätze wuchsen um $5,4 \%$ statt $4,2 \%$ geplante, die öf entlichen Investitionen wurden mit einem Wachstum von $17 \%$ um $3 \%$ übertroffen, die privaten Investitionen mit $13,6 \%$ um $0,6 \%$.

29 Berechnet nach Presidencia, (Fn. 12), S. 369, 541, 573.

30 PND, (Fn. 19), S. 19; Presidencia (Fn. 12), S. 582; Financial Times, 7. 7. 1981.

31 Vgl. PND, (Fn. 19), S. 19; Secretaría de Programación y Presupuesto, Informe sobre la Situación Economica, in: Uno más uno, 18. 4. 1983, S. 16. 
wird für 1983 mit einem Uberschuß von 10 mrd US \$ gerechnet; die ausländische Verschuldung soll sich gegenüber 1982 lediglich um 5 mrd US $\$$ ausdehnen. Dem steht allerdings gegenüber, daß sich das BIP um ca. $5 \%$ vermindern wird, daß die Investitionsrate den niedrigsten Stand seit 50 Jahren erreicht hat, und daß die Mindestlöhne, von denen etwa $60 \%$ der Arbeiter abhängig sind, Mitte des Jahres lediglich um $15 \%$ angehoben wurden. Während sich 1982 die Arbeitsplätze um 0,8\% verringerten, wird damit gerechnet, daß bis 1985 weitere 1,5 mio Arbeitsplätze verloren gehen. Dies entspricht der Hälfte der Arbeitsstellen, die zwischen 1977 und 1981 geschaffen wurden..$^{32}$ Damit sind die größten Teile der Bevölkerung an den Rand der Existenz gedrängt. Die inflationäre Tendenz der mexikanischen Okonomie wurde, zumindest ab 1979, über eine zu hohe Nachfrage bei einem sich nicht schnell genug ausdehnenden Angebot erklärt. Die überhöhte Nachfrage wurde hauptsächlich durch höhere Staatsausgaben iniziiert, die über höhere Verschuldung finanziert wurde. Eng damit verbunden war ein über 6 Jahre hinweg durchschnittlich um $30 \%$ gestiegener Geldumlauf. ${ }^{33}$ Noch Anfang 1981 meinte der damalige Planungsminister und heutige Staatspräsident de la Madrid Hurtado, die inflationären Tendenzen müßten mit höheren Staatsausgaben bekämpft werden, indem in Bereiche mit mangelndem Angebot investiert wird. ${ }^{34}$ Die Regierung überschätzte dabei bei weitem die Anpassungsstrukturen des Produktionsapparates.

Ein weiterer schwerwiegender Fehler der Wirtschaftspolitik war die Uberbewertung des Peso, der von 1977 bis Anfang 1982 gegenüber dem Dollar auf gleicher Höhe gehalten wurde. Dabei wurde die ohnehin niedrige internationale Konkurrenzfähigkeit der mexikanischen Industrie weiter geschwächt. Vor allem aber waren bei gestiegener Inflation und bei erhöhten inländischen Zinssätzen die Flucht in den US Dollar die preisgünstigste Möglichkeit, Kapital zu erwerben und Ersparnisse in Sicherheit zu bringen. ${ }^{35}$ Die überraschende Geschwindigkeit und das Ausmaß des Zusammenbruchs der mexikanischen Ókonomie 1982 sind zu einem großen Teil auf die falschen Maßnahmen der früheren Regierung zurückzuführen. Neben der Erklärung über eine verfehlte Wirtschaftspolitik und eine blinde Euphorie, in der gigantische Projekte möglichst schnell verwirklicht werden müssen, ${ }^{36}$ hat die Uberbewertung des Peso auch einen rationalen

32 Presidencia, (Fn. 12), S. 573; Uno más uno, 28. 7. 1983, S. 8; 2. 9. 1983, S. 9; 11. 4. 1983, S. 7; Excelsior, 2. 7. 1983, S. F-1; Proceso, (336), 11. 4. 1983, S. 8.

33 Ähnliche Inflationsanalysen: Clavijo, F., Reflexiones en torno a la inflación mexicana, in: Trimestre Económico, (47-4), 1980, S. 1048; Yacamán, J. M., Análisis de la inflación en México, in: Trimestre Económico, (50-3), 1983, S. 1572; Pazos, L., La estatización de la banca, Hacia un capitalismo de estado?, México 1982, S. 37: PND, (Fn. 19), S. 100; Internationaler Währungsfond: Excelsior, 27. 4. 1983, S. A-7. Dagegen: Aceituno, G./Ruprah, I., Déficit público e inflación, in: CIDE, (Fn. 28).

34 Secretaría de Programación y Presupuesto, Memoria Institucional 1981, México 1982, S. 374.

35 Zur Erklärung der mexikanischen Krise vgl. auch: Street, J. H., Coping with Energy Shocks in Latin America: Three Responses, in: Latin American Research Review, (17-3), 1982, S. 144; Rivera Rios, M. A., México: devaluación y crisis, in: Teoría y Política, (3-7/8), 1982, S. 71. Die Verteidigung der staatlichen Expansionspolitik: Montemayor, R., El diseño de una estrategía de desarrollo, in: González M, H. E., El sistema económico mexicano, México 1982, S. 26: Villarreal, R., Petroleum and Mexican Economic Growth and Development in the 1980s, in: Ladman, (Fn. 3).

36 Dazu vor allem Kaplan, M., Petróleo y desarrollo: El impacto interno, in: Foro Internacional, (21-1), 1980, S. 90-93. 
Grund. Sowohl 1976 als auch 1982 wurde erst kurz vor oder bereits nach dem Regierungswechsel stark abgewertet. Damit können die Gelder aus der, zum Ende einer Regierungsperiode, stark steigenden Korruption zu günstigen Kursen ins Ausland transferiert werden.

\section{Grundstrukturen der mexikanischen Ökonomie}

Aus der Beschreibung des Zustandekommens der Inflation ist noch nicht ohne weiteres ersichtlich, weshalb daraus Rezession resultiert. Dazu reicht meines Erachtens eine bloße Untersuchung monetärer Strategien, etwa der Staatsverschuldung und der Ausweitung des Geldumlaufs, nicht aus. Vielmehr ist dazu eine Analyse des Produktionsund Distributionsapparates notwendig. Es bleibt zu fragen, wieso der Produktionsapparat nicht flexibel auf die erhöhte Nachfrage reagieren kann, wie im vorigen Abschnitt behauptet wurde. Eine kritische Untersuchung dazu bietet die neue Regierung selbst. Im nationalen Entwicklungsplan von 1983 wird das Scheitern der Erdölstrategie hauptsächlich über "strukturelle Probleme der Okonomie" erklärt: Ungleichgewichte zwischen den verschiedenen Sektoren haben seit 1965 insbesondere den Agrarsektor benachteiligt. Selbst die verstaatlichten Wirtschaftsbetriebe haben teilweise einer Integration des Produktionsapparates entgegengewirkt. Die ungleiche Einkommenstruktur hat sich kaum verändert, wobei die Konsumstruktur der Reichen, die sich auf kapitalintensive und importierte Güter konzentriert, die Desintegration verstärkt. Bereits 1976 bestand diese strukturelle Krise und konnte lediglich durch den Olboom überdeckt werden. Zur Uberwindung der Krise ist deshalb zunächst eine kurzfristige Bekämpfung der Inflation und des externen Ungleichgewichts nötig, längerfristig sind aber qualitative Veränderungen unumgänglich. ${ }^{37}$

Welche ökonomischen Mechanismen aber diese Ungleichgewichte hervorruferı, und was letztlich die Grundmerkmale eines desintegrierten Produktionsapparates ausmacht, wird im nationalen Entwicklungsplan nicht hinterfragt. Die hier zu entwickelnde Analyse wird in ihrer daraus resultierenden Strategie deshalb auch von den Veränderungsvorstellungen des Entwicklungsplanes abweichen.

Die austeritäre Politik der neuen Regierung bei anhaltend hoher Inflation senkt nicht nur das Realeinkommen der unteren und mittleren Gesellschaftsschichten auf ein bedrohliches Niveau ab, sondern es erhöht sich dadurch auch die Einkommenungleichheit. Während die Bezieher von Minimallöhnen allein in den ersten 9 Monaten 1983 etwa $45 \%$ ihres Realeinkommens verloren, halten die Unternehmer durch steigende

37 PND, (Fn. 19), S. 89-116. Der Monetarist Pazos, L., Respuesta a los planes de MMH, México 1983, S. 101 kommentiert auch prompt, daß der Begriff "qualitative Veränderung" der marxistischen Ideologie entspringe. In der Tat steckt hinter dieser Krisenanalyse das Konzept der "strukturellen Heterogenität", wie es von Dependenztheoretikern und Marxisten hervorgebracht wird. Der mexikanische Staat ist also flexibel genug auf eine Analyse der Linken eine ganz andere Strategie auf zubauen. 
Preise zumindest ihre Verluste in Grenzen. Darüberhinaus erlaubten sich die oberen Chargen der Bürokratie Gehaltserhöhungen von $80 \%-160 \%{ }^{38}$ Eine sehr starke ungleiche Verteilung der Einkommen ist ein Phänomen, das die mexikanische Gesellschaft seit jeher durchzieht und auch durch die Revolution kaum verändert wurde. Eine Analyse der zugänglichen Verteilungsdaten ergibt, daß die untersten $40 \%$ ihren Anteil am Volkseinkommen von $13 \% 1950$ auf $11 \% 1977$ abgesenkt haben. Die oberen $40 \%$ hielten ihren Anteil am Gesamteinkommen bei etwa $76 \%$. Innerhalb dieser Gruppe ergaben sich allerdings Veränderungen. Die obersten $20 \%$ der Einkommen sanken von $59 \% 1950$ auf $55 \%$ 1977, wobei die der nächsthöheren $20 \%$ von $17 \%$ auf $21 \%$ stiegen. $^{39}$ Das läßt darauf schließen, daß sich die Mittelschichten einen etwas höheren Anteil am Volkseinkommen sichern konnten, während die untersten Schichten abfielen. Im Vergleich zu anderen Entwicklungsländern ist die Einkommensverteilung in Mexiko hoch konzentriert. ${ }^{40}$ Trotz hoher Wachstumsraten seit den 1940er Jahren und einer stetig steigenden Industrialisierung haben sich Umverteilungsprozesse, wie sie bei der Entwicklung kapitalistischer Industrieländer beobachtet wurden, nicht ergeben. Auch wenn die mangelnde Befriedigung der Grundbedürfnisse großer Bevölkerungsteile betrachtet wird, muß ein Durchsickern des Wohlstandswachstums auf die ärmeren Schichten der Bevölkerung verneint werden. ${ }^{41}$

Bei der Erklärung der ungleichen Einkommensverteilung spielt die fehlende Durchsetzung höherer Löhne eine wichtige Rolle. Da etwa 50 \% der Erwerbstätigen arbeitslos oder unterbeschäftigt sind, ${ }^{42}$ kann die Arbeitslosigkeit selbst in einer Wachstumsphase, wie zwischen 1979 und 1981, kaum verringert werden. Durch das Uberangebot unqualifizierter Arbeitskraft können von Arbeitnehmerseite her keine höheren Löhne durchgesetzt werden, die dem durchschnittlichen Produktivitätsfortschritt entsprächen. Daß

38 Proceso, (347), 28. 6. 1983, S. 22. Im Juni 1983 wurden die Mindestlöhne um 15 \% erhöht, während die Inflation bis September auf $60 \%$ gestiegen war.

39 SPP, (Fn. 34), Anexo I, S. 33. Bezeichnenderweise liegen seit 1977 keine Verteilungszahlen mehr vor. Seit der Krise hat sich die Einkommensverteilung mit Sicherheit noch verschlechtert, zumal auch im Olboom die Lohnsteigerungen hinter den Wachstumsraten blieben. Von Oktober 1976 bis Mai 1983 fiel der reale Mindestlohn um $45 \%$. Zahlen der Banco de México, in: Uno más uno, 3. 6. 1983, S. 8.

40 In einer Untersuchung über 47 Entwicklungsländer stand Mexiko Anfang der 70er Jahre an 6. Stelle der Einkommenskonzentration bezüglich der $20 \%$ höchsten Einkommen. Ahluwalia, M. S., Income Distribution: Some Dimensions of the Problem, in: Chenery, H. B., et al., Redistribution with Growth, London 1974, S. 8-9. Nach Nicaragua, Haiti und Guatemala wies Mexiko 1976 die höchste Einkommenskonzentration Lateinamerikas auf. Ehrke, M., Mexikanische Erdölpolitik im Rahmen der Beziehung Mexiko-USA, Bonn 1979, S. 34.

41 Ca. $40 \%$ der Mexikaner leben unterhalb der Armutsgrenze, ca. $50 \%$ sind unterernährt. Vgl. Molina S, S., La probreza, Descripción y análisis de políticas para superarla, in: Revista de la CEPAL, (18), 1982, S. 95; Bergsman, J., Income Distribution and Poverty in Mexico, World Bank, Working Paper No. 395, Washington 1980, S. 21-23; PND, (Fn. 19), S. 228; Proceso, (340), 9. 5. 1983, S. 7.

421983 waren 2 mio arbeitslos, 10 mio. unterbeschäftigt, was ca. $50 \%$ aller ökonomisch Aktiven entspricht. Dieses Verhältnis hat sich seit 1970 auch nicht verändert. Harris, R. L., The Political Economy of Mexico in the Eighties, in: Latin American Perspectives, (9-1), 1982, S. 3; Rott, R., Industrialisierung und Arbeitsmarkt, Aspekte der sozioökonomischen Entwicklung in Kolumbien und Mexiko, Königstein 1979, S. 423; Trejo Delarbre, R., El movimiento obrero, in: González Casanova, P./Florescano, E., México hoy, México 1979, S. 122. Zahlen der Bancomer, in: Uno más Uno, 3. 6. 1983, S. 8. 
unqualifizierte Arbeit auch außerhalb von Lohnverhältnissen ihr Einkommen nicht erhöhen kann, zeigt sich daran, daß nur $43 \%$ der Einkommen der ärmsten $20 \%$ der Bevölkerung aus Lohneinkommen, $45 \%$ aber aus selbständiger Arbeit erzielt werden. ${ }^{43}$ Eine Erhöhung der Löhne ist allerdings den Arbeitern in jenen Sektoren möglich, in denen Mangel an qualifizierter Arbeitskraft besteht und/oder in denen ein hoher Organisationsgrad die Konkurrenz auf dem Arbeitsmarkt beseitigt, wie das Pemex-Beispiel zeigt. Dies führt zu einer heterogenen Struktur der Lohneinkommen, wobei die ungleiche Einkommensverteilung auch mit durch die ungleichen Arbeitereinkommen begründet ist.

Wie am Beispiel der Entwicklung der kapitalistischen Entwicklungsländer gezeigt werden kann, ist eine relativ egalitäre Einkommensverteilung zumindest ab einem gewissen Entwicklungsgrad eine Voraussetzung für kapitalistisches Wachstum und nicht nur eine mögliche Folge. Die Beschränkung einer Entwicklungsstrategie auf Industrialisierung und Durchkapitalisierung mit hohen Wachstumsraten und damit hohen Investitionsmöglichkeiten reicht nicht aus, wenn erst im weiteren Verlauf Verteilungseffekte erwartet werden, zunächst aber hohe soziale Kosten in Kauf genommen werden müssen. Vielmehr scheint als weitere Grundlage die Ausweitung der internen kaufkräftigen Massennachfrage ausschlaggebend zu sein. Innerhalb dieses Paradigmas wird die Verbindung von gesteigerter Produktivität und zusätzlicher Mobilisierung von Arbeitskraft und damit einhergehende Umverteilung von Einkommen zum grundlegenden Mechanismus eines ökonomischen Wachstumsprozesses. ${ }^{44}$

$\mathrm{Da}$ in Mexiko die Anbieter unqualifizierter Arbeit ihre Einkommen nicht erhöhen können, ergibt sich folgender Mechanismus, der zu ökonomischer Ungleichgewichtigkeit führt: Die Bezieher niedriger Einkommen arbeiten in Sektoren mit niedriger

43 Ein Sachverhalt, der zunächst noch paradoxer erscheint, wenn man hinzufügt, daß das Einkommen der $10 \%$ Reichsten zu $68 \%$ aus abhängiger und nur zu $22 \%$ aus selbständiger Arbeit stammt. Berechnet aus: Hernandez Laos, E./Córdova, J., La distribución del ingreso en México, México 1982, S. 80-81.

44 Da diese zentrale These hier nicht weiter ausgeführt werden kann, soll ein etwas breiterer Literaturhinweis gegeben werden. Umverteilung als Grundlage von Wachstum: Elsenhans, H., Rising Mass Income as a Condition of Capitalist Growth: Implications for the World Economy, in: International Organization, (37-1), 1983, S. 5-12; Senghaas, D., Von Europa lernen, Entwicklungstheoretische Betrachtungen, Frankfurt 1982, S. 75. Bloße Einkommensumverteilung nützt nichts, sie muß mit einer Mobilisierung von Arbeitskraft verbunden sein: Elsenhans, H., Die Úberwindung von Unterentwicklung durch Massenproduktion für den Massenmarkt, in: Handbuch der Dritten Welt, Bd. 1, Unterentwicklung und Entwicklung, Hamburg 1982; Ahluwalia, M. S./Chenery, H., The Economic Framework, in: Chenery, et al., (Fn. 40), S. 43. Hohe Löhne notwendig für Massenproduktion: Strassmann, W. P., Economic Growth and Income Distribution, in: Quarterly Journal of Economics, (70-3), 1956, S. 430. Wachstum bringt nicht automatisch Verteilung, sondern muß politisch durchgesetzt werden: Cline, W. R., Potential Effects of Income Redistribution on Economic Growth, Latin American Cases, New York 1972, S. 8; Singer, H. W., Poverty, Income Distribution and Levels of Living, in: Rao, C. H. H./Joshi, P. C., Reflections on Economic Development and Social Change, Oxford 1979, S. 32; Burki, S. J./Ul Haq, M., Meeting Basic Needs: An Overview, in: World Development, (9-2), 1981, S. 174; Chenery, H., Armut und Fortschritt - Alternativen für die Dritte Welt, in: Finanzierung und Entwicklung, (17-2), 1980, S. 13. Wirkungen höherer Verteilung in Mexico: Stewart, J. R., Potential Effects of Income Redistribution on Economic Growth: Mexico, in: Economic Development and Cultural Change, (26-3), 1978, S. 484; Ginneken, W., Socio-Economic Groups and Income Distribution in Mexico, in: International Labour Review, (118-3), 1979, S. 341. 
Produktivität, hauptsächlich im Agrarbereich, im städtischen informellen Sektor und in Teilen des Dienstleistungsbereichs. Da diese Arbeitskraft billig bleibt, besteht auch keine direkte Notwendigkeit ihre Produktivität zu erhöhen. In kapital- und qualifikationsintensiven Branchen ergibt sich dagegen eine wesentlich höhere und steigende Produktivität. Zwischen dem Agrar- und Industriesektor besteht ein Unterschied der Arbeitsproduktivität von 1:5,7, der sich stetig erhöht. ${ }^{45}$ Da aber "Produktivität" im Vergleich zwischen verschiedenen Sektoren nur über Preise gemessen werden kann und nicht etwa als reale output Menge, zeigt der Produktivitätsunterschied lediglich eine relative Uberbewertung der Produkte der "produktiven" Sektoren an. Für Produkte aus Branchen, die mit hoher Kapitalintensität und besser bezahlter qualifizierter Arbeit hergestellt werden, steigen die Preise also tendenziell gegenüber den Produkten und Dienstleistung, die mit geringer Arbeitsentlohnung hergestellt werden. ${ }^{46}$ Innovationsfortschritte der Produktion werden aufgrund dieser Struktur nicht an die Masse der Bevölkerung in Form höherer Löhne weitergegeben, wie dies in entwickelten kapitalistischen Ókonomien der Fall ist. Dort werden Produktivitätsfortschritte auch nur in einigen Branchen erzielt, aufgrund der Knappheit und der Mobilität von Arbeitskraft steigt das Lohnniveau aber in der gesamten Okonomie. Da die Preise der Güter aus den "unterproduktiven" Sektoren in Mexiko im Verhältnis zu den anderen zu niedrig liegen, bringen folglich Investitionen nur unterdurchschnittliche Profite, selbst wenn in diesen Sektoren Mehrwert erzeugt wird. In einem solchen Zirkel der Blockierung entsteht keine zusätzliche kaufkräftige Nachfrage und es wird nicht für den Massenkonsum produziert, sondern nur für kleine Märkte des Luxuskonsums privilegierter Schichten.

Durch die Beschreibung mangelnder Profitabilität und zu geringem Innovationsdrucks in Sektoren mit zu billiger Arbeitskraft, können viele Beobachtungen "struktureller Heterogenität" meines Erachtens erst erklärt werden: Seit 1940 war ein hohes ökonomisches Wachstum ein Grundmerkmal der mexikanischen Entwicklung. Von 1950 bis 1959 betrug das durchschnittliche jährliche Wachstum 6,3\%, von 1960 bis 1969 7,1 \% und von 1970 bis 1979 5,6\%.47 Trotz einer umfangreichen Agrarreform, in deren Verlauf über $50 \%$ der Agrarfläche verteilt wurde, konzentrierte sich der Landbesitz, die Produktion und die Produktivität erneut. Die Heterogenisierung innerhalb des Agrarsektors wurde dadurch gefördert, daß Neolatifundisten in Zusammenarbeit mit dem Agrobusiness verstärkt hochwertige Exportprodukte für den US-Markt herstellen, während kleine Produzenten für den internen Markt Grundnahrungsmittel anbauen, deren Preise künstlich auf niedrigem Niveau gehalten werden. Zu diesem Zweck wurde die staatliche Ankauf- und Verteilungsorganisation CONASUPO aufgebaut. Es waren somit die

45 NAFINSA, La economía mexicana en cifras, México 1981, S. 42. Zahlen für 1979; 1970 betrug das Verhältnis noch $1: 4,8$.

46 Abgesehen davon produzieren die Bereiche mit höherer Kapitalintensität und teurerer Arbeitskraft natürlich auch effizienter, was bei steigenden Preisen/Löhnen sicherlich auch in den unterproduktiven Sektoren geschehen würde. Vgl. dazu Elsenhans, H., Abhängiger Kapitalismus oder bürokratische Entwicklungsgesellschaft, Versuch über den Staat in der Dritten Welt, Frankfurt 1981, S. 44.

47 NAFINSA, (Fn. 45), S. 21. 
kleinen Agrarproduzenten, die über niedrige Preise zum Industriewachstum beitrugen. Seit 1965 sank die Grundnahrungsmittelproduktion beständig ab, da die Bauern nicht zu einer höheren Produktion bereit waren. Dies wiederum trug zur unterschiedlichen Produktivität zwischen Agrar- und Industriesektor bei. ${ }^{48}$

Bei einem Wachstumsprozeß, mit dem nicht gleichzeitig Umverteilungsprozesse verbunden sind, kommt es zu einer Auseinanderentwicklung der verschiedenen Sektoren. Da die Einkommensunterschiede weit größer sind, als durch unterschiedliche Qualifikationen gerechtfertigt wäre, verzerrt sich die gesamte Preisstruktur. Umfangreiche staatliche Preissubventionen, die sich von 3,6\% auf 13,4\% am BIP von 1970 bis 1981 erhöhten, ${ }^{49}$ verzerren die Preisstruktur weiter. Bei niedrigen Einkommen werden Subventionen für Grundnahrungsmittel, Massentransport, Elektrizität usw. für die unteren Schichten zur Existenznotwendigkeit, direkt einkommenswirksame Transfers würden aber verhindern, daß gut verdienende Schichten an verbilligten Gütern partizipieren und die Kluft zwischen Ausgaben für das Existenzminimum und den Konsumstrukturen der Mittel- und Oberschicht sich weiter vergrößert.

Mitzieheffekte, die sich durch Umverteilung von Produktivitätsfortschritten durch steigende Preise auch in den übrigen Sektoren niederschlagen, ergeben sich nicht. Da dann wesentlich höhere Profite in den Sektoren erzielt werden, die für den gehobenen Anspruch produzieren, wird die Investition in die anderen Sektoren vernachlässigt. Aus diesem Sachverhalt erklärt sich auch die Beobachtung, daß die Bourgeoisie nicht in die unrentablen Produktionssektoren investiert, sondern eher zu Spekulationsgeschäften neigt, bzw. Kapital ins Ausland transferiert. Durch die zunehmende Arbeitsteilung und Abgrenzung der Aktivitäten im Industriesektor zwischen den Multinationalen Konzernen, dem nationalen Kapital und den staatlichen Betrieben, hat sich in vielen Bereichen eine monopolistische Situation ergeben, in der Profiterhöhung über Güterverknappung und nicht über innovative Fortschritte erzielt werden. ${ }^{50}$

48 Vgl. Stavenhagen, R., Capitalism and the Peasantry in Mexico, in: Latin American Perspectives, (5-3), 1978, S. 33; Bartra, R., Und wenn die Bauern verschwinden . . Uberlegungen zur politischen Konjunktur in Mexiko, in: Lateinamerika Analysen und Berichte 1, Berlin 1977, S. 120; Steger, H.-A., Participación política y resistencia cultural en México, in: Khipu, (3-5), 1980, S. 41; Nohlen/Lehr, (Fn. 17), S. 128; Gomez Oliver, L., Crisis agricola, crisis de los campesinos, in: Comercio Exterior, (28-6), 1978, S. 724; Ngyen, D. T./Martinez-Saldivar, M. L., The Effects of Land Reform on Agricultural Production, Employment and Income Distribution, A Statistical Study of Mexican States, 1959-1969, in: Economic Journal, (89-355), 1979, S. 634.

49 Vgl. Dávila Jiménez, G., La política de precios y subsidios, in: González, (Fn. 35), S. 243; Méndez Morales, J. S., Cien preguntas y respuestas en torno a la exonomía mexicana, México 1983, S. 73.

50 Casar, J., Ciclos económicos en la industria y sustitución de importaciones: 1950-1980, in: CIDE, (Fn. 28), S. 95. Pazos, (Fn. 33) errechnet, daß von 1980 bis 1982 etwa 50 mrd US $\$$ ins Ausland abgeflossen sind, was $2 / 3$ der mexikanischen Auslandsverschuldung entspricht. Selbst in der offiziellen Statistik werden Kapitalabflüsse von 17 mrd US \& für diesen Zeitraum angegeben. Presidencia, (Fn. 12), S. 574. Zur Industriekonzentration: Novelo Novelo, E., La estructura económica de las 500 empresas más grandes de México, México 1982, S. 114. 


\section{Die Funktion des Staates}

In dieses Szenario einer zunehmend sich heterogenisierenden Ókonomie greift der mexikanische Staat immer stärker ein. In einem Zeitraum von 10 Jahren haben sich die Ausgaben der föderalen Regierung am BIP von 28,1 \% 1973 auf 59,5 \% 1982 erhöht, wobei der Gesamtanteil des Staates $67 \%$ beträgt. ${ }^{51}$ Damit hat Mexiko die Staatsquote entwickelter kapitalistischer Länder, die zwischen 40 und $50 \%$ liegt, bei weitem überschritten. Die Steuereinnahmen der föderalen Regierung am BIP konnten zwar von 10,1\% 1973 auf 16,9\% 1982 erhöht werden, das Defizit des öffentlichen Sektors am BIP erhöhte sich aber wesentlich stärker von $4,8 \%$ auf $17,7 \% . .^{52}$

Der staatliche Einfluß dehnte sich ebenfalls im Produktionsbereich aus. Die Investitionen der föderalen Regierung einschließlich der öffentlichen Unternehmen erhöhten sich von 7,2 \% am BIP 1973 auf 12,8 \% 1981, wobei der Anteil der öffentlichen Investitionen, der im industriellen Sektor getätigt wird, von $32 \%$ auf $50 \%$ stieg. In 376 Industrieunternehmen produzierte der Staat $198232 \%$ des Industrieprodukts. 1981 waren von den 20 größten Unternehmen Mexikos 9 staatlich. ${ }^{53}$ Neben dem hohen Einfluß in der Industrie übernahm der Staat durch die Bankenverstaatlichung im September 1982 den gesamten Finanzsektor, an dem er zuvor schon mit ca. $50 \%$ beteiligt war. $^{54}$

Die verstärkten Eingriffe des Staates in die Okonomie setzten ein, als das Scheitern der Importsubstitutionsphase Ende der 1960er Jahre offensichtlich wurde. Nach anfänglichen Erfolgen in der Diversifizierung der Produktionsstruktur durch die seit 1940 betriebene Protektionspolitik, bildeten sich die oben beschriebenen Charakteristiken der mexikanischen Okonomie heraus. ${ }^{55}$ Ab 1970 reagierte der mexikanische Staat periodisch mit einer Wirtschaftspolitik keynesianistischer Ankurbelung und monetaristischer Konsolidierungsphasen. Dies erklärt sich dadurch, daß sich mit den verstärkten Staateingriffen ökonomische Zyklen ergeben, die sehr stark durch die sechsjährigen Regierungsperioden - sexenios - beeinflußt sind. Da Mexiko zwar seit über 50 Jahren von derselben Partei regiert wird, der Präsident aber nicht wiederwählbar ist, ergeben sich bei Amtsantritt regelmäßig Umorientierungsphasen, in denen praktisch alle wichtigen

51 Berechnet nach Presidencia, (Fn. 12); Excelsior, 14. 5. 1983, S. A-1. In den 1950er Jahren lag der Staatsanteil am BIP noch bei 13-15\%, in den 60er Jahren bei 20-26\%. Carmona, F., Estado y capitalismo en México: imbricación creciente con la sociedad civil, in: Alonso, J., El Estado mexicano, México 1982, S. 34.

52 Berechnet nach Presidencia, (Fn. 12). Vgl. zum gestiegenen Steueraufkommen auch: Evans, J. S., The Evolution of the Mexican Tax System Since 1970, Institute of Latin American Studies, Papers no. 34, Austin 1982.

53 Berechnet nach: Presidencia, (Fn. 12), S. 450; Novelo Novelo, (Fn. 50), S. 18; Excelsior, 5. 8. 1983, S. A-4. Vgl. auch: Carrillo Castro, A., La reforma administrativa en México, México 1980.

54 Der Staat kam dadurch in den Besitz der Aktiva von 37 Privatbanken im Wert von 3,3 bio. Pesos (ca. 50 mrd US \$) und wurde gleichzeitig Eigentümer derer Industrieaktien. Pazos, (Fn. 33), S. 44.

$55 \mathrm{Vgl}$. FitzGerald, E. V. K., A Note on Capital Accumulation in Mexico, in: Development and Change, (11-3), 1980, S. 392; Mueller-Ohlsen, L., Importsubstitution und Exportdiversifizierung im Industrialisierungsprozeß Mexikos, Tübingen 1974, S. 171; Ramírez, R., Industrialización y sustitución de importaciones en México, in: Comercio Exterior, (30-1), 1980, S. 32. 
Posten in der Regierung, der Bürokratie und den staatlichen Unternehmen umbesetzt werden. In einer zweiten Phase nimmt die staatliche Investition zu und ruft zunächst, wie zwischen 1972-1974 und 1979-1981, Wachstumsphasen hervor. Da der heterogene und ineffiziente Produktionsapparat aber nicht flexibel auf die verstärkte staatliche Nachfrage reagieren kann, entstehen Engpässe, auf die Inflation und Handelsbilanzdefizite folgen. Der Staat muß darauf wiederum mit einer restriktiven Wirtschaftspolitik reagieren. Diese Phase trifft etwa die letzten 2 Jahre eines sexenios, die zudem von internen Auseinandersetzungen um die neue Präsidentschaft geprägt sind. ${ }^{56}$

Neben den stärkeren direkten staatlichen Eingriffen stand als weiteres Merkmal der mexikanischen Wirtschaftspolitik die Wachstumsförderung und damit die Unterstützung der Bourgeoisie im Vordergrund. Wie in der gesamten Entwicklung Lateinamerikas wird der Bourgeoisie auch in Mexiko weder eine dominante noch eine progressive Rolle zugeschrieben. Der revolutionäre Staat nach 1924 hat seine Bourgeoisie erst "gemacht", und nicht umgekehrt. Allerdings hat der Staat diese Bourgeoisie ab 1940 sehr stark gestützt und gefördert. Die Bourgeoisie wurde dadurch mehr und mehr zur stärksten gesellschaftlichen Kraft außerhalb des Staates, hat sich teilweise von ihm gelöst und sich emanzipiert. ${ }^{57}$ Die Bourgeoisie und den Staat in Mexiko verbinden gemeinsame Interessen. Nur durch ökonomisches Wachstum kann das Profitinteresse des privaten Kapitals befriedigt werden. Zum andern braucht auch der Staat ein wachsendes Sozialprodukt um seine Ausdehnung finanzieren zu können. Der Regierung ist dabei bewußt, daß sie eine Wachstumsstrategie effizienter über die kooperierende Investitionstätigkeit der Privaten erreicht. Ein weiteres gemeinsames Interesse besteht darin, die Wachstumserfolge durch die Dominanz der Massen nicht allzu durchgreifend verteilen zu müssen, dabei aber das System stabil zu halten. Es wird deshalb zurecht von einer "Allianz für Profite" gesprochen. ${ }^{58}$

Die Möglichkeit des Staates zu seiner ökonomischen Ausdehnung lassen sich hauptsächlich durch seine starke politische Stellung und die damit verbundene relative Schwäche der übrigen gesellschaftlichen Gruppen erklären. Nur daraus, daß der mexikanische Staat eine Politik betreibt, die häufig im Interesse der Bourgeoisie liegt, kann noch nicht gefolgert werden, daß das private Kapital dominiert. Ebensowenig stützt der Staat die

56 Ehrke, (Fn. 25), S. 194: Ehrke, M., Mexiko: Der Weg in die Krise, Hamburg 1983, im Erscheinen.

57 Whitehead, L., Por que México es casi ingobernable, in: Revista Mexicana de Sociología, (42-1), 1980, S. 856; Nohlen/Lehr, (Fn. 17), S. 133; Rott, R., Die mexikanische Gewerkschaftsbewegung, Kronberg 1975, S. 299: Vernon, R., The Dilemma of Mexico's Development, Cambridge 1963, S. 9; González Casanova, P., Las alternativas de la democracia, in: González Casanova/Florescano, (Fn. 42), S. 406. Hansen, R. D., The Politics of Mexican Development, Baltimore 1971, S. 87: Kaum ein lateinamerikanisches politisches System hat seine Bourgeoisie so stark gestützt, wie Mexiko.

58 Kaufman Purcell, S., Business - Government Relations in Mexico, the Case of the Sugar Industry, in: Comparative Politics, (13-2), 1981, S. 211; Weinert, R. S., The State and Foreign Capital, in: Reya, J. S./Weinert, R. S., Authoritarianism in Mexico, Philadelphia 1977, S. 124: Bennett, D./Sharpe, K., The State as Banker and Entrepreuner, in: Comparative Politics, (12-2), 1980, S. 180; Smith, P. H.: Does Mexico Have a Power Elite?, in: Reyna/Weinert, (Fn. 58), S. 147; Stevens, E. P., Mexicos One-Party State, in: Wiarda, H. J./Kline, H. F., Latin American Politics and Development, Boston 1980, S. 419. 
Bourgeoisie um jeden Preis. Dies geschieht nur, solange gemeinsame Interessen bestehen. In der Auseinandersetzung mit dem privaten Kapital hat der Staat wesentlich druckvollere Mittel zur Hand. Diese reichen von der politischen Mobilisierung der gesellschaftlichen Sektoren, insbesondere der Gewerkschaften, bis zu ökonomischen Maßnahmen durch Festsetzung von Preissystemen und der inzwischen monopolistischen Vergabe von Kreditmitteln. Neben verbalradikalen ideologischen Offensiven in der Offentlichkeit bleiben den Unternehmern nur die Investitionsverweigerung und die Kapitalflucht ins Ausland als passive Reaktionsmöglichkeiten. ${ }^{59}$ Mit einer Verfassungsänderung Anfang 1983 hat die neue Regierung dem absoluten Führungsanspruch in der Okonomie auch eine rechtliche Grundlage hinzugefügt. In einer gemischten Ökonomie economía mixta - kommt dem Staat die Lenkung - rectoría del Estado - der Entwicklung zu. Der Präsident sieht die Führung des Staates als eine der Grundprinzipien der mexikanischen Gesellschaftsstruktur. Mit einer gleichzeitig höheren Sozialpflichtigkeit des Privateigentums wird dieses vermehrt zu einer Konzession des Staates, wobei es seit 1917 auch nicht als ein Grundrecht anerkannt ist. ${ }^{60}$

Neben einer relativen Dominanz über die Bourgeoisie hat sich auch eine absolute und institutionalisierte Dominanz des Staates über die übrigen gesellschaftlichen Gruppen ergeben. Das durch die Revolution von 1917 entstandene Machtvakuum konnten weder die Bauern, die zu großen Teilen die Revolution trugen, noch die zahlenmäßig sehr kleine Arbeiterklasse, noch die schwache Bourgeoisie füllen. Vielmehr übernahmen die Revolutionsgeneräle, die an keiner der anderen gesellschaftlichen Gruppen explizit orientiert waren, unter heftigen internen Kämpfen die Macht. Trotz der Konflikte innerhalb der Gruppe der militärischen Führer gelang die Konsolidierung der Macht in der Form eines starken und zentralisierten Einparteiensystems. Unter der Reformorientierung von Cárdenas (1934-1940) nahm das politische System seine bis heute bestehende Form an. Die Partido Revolucionario Institucional - PRI - wurde zum Instrument der Integration und der Kontrolle der Massen. Cárdenas' Reformvorstellungen in einem populistischen Bündnis verkehrten sich in der weiteren Entwicklung. Anstelle der Partizipation der Massen trat die wirksame Einbindung und Kontrolle breiter Schichten in den Staatsapparat durch staatliche Gewerkschaften, staatliche Bauernverbände und die Organisation der Mittelschichten innerhalb der PRI. Durch die Machtkonzentration im Staatsapparat wurde die populistische Herrschaftsausübung zu einem einseitigen Instrument der Massenmobilisierung und Massenintegration, die durch die revolutionäre Ideologie verschleiert wird. Tatsächlich entstand ein autoritäres Regime. Die sehr flexible Anwendung von Integrationsmechanismen in Form der Kooptation von

59 Cosío Villegas, D., El sistema político mexicano, México 1972, S. 72; Whitehead, (Fn. 57), S. 856; Mols, M., Mexiko im 20. Jahrhundert, Paderborn 1981, 314; Arriola, C., Los grupos empresariales frente al Estado, in: Las fronteras del control del Estado mexicano, México 1976, S. 56, 70; Eckstein, S., Politicos and Priests, in: Comparative Politics, (9-4), 1977, S. 417.

60 Diario Oficial, 3. 2. 1983. Vor allem Art. 25. De la Madrid Hurtado, M., Primer Informe de Gobierno 1983, in: Uno más uno, suplemento, 2. 9. 1983, S. 2. 
Regimegegnern und andererseits von Repression erklärt die politische Stabilität über nunmehr 50 Jahre. ${ }^{61}$

Aufgrund der unkontrollierten politischen und ökonomischen Stärke des Staates, kann durchaus von einer eigenen Klasse, einer Staatsklasse, gesprochen werden. ${ }^{62}$ Der Staat bleibt dabei nicht bloßes Instrument einer gesellschaftlichen Gruppe oder Klasse, durch das regiert wird, sondern er wird zur eigenständigen dominanten gesellschaftlichen Kraft. Bei der Untersuchung der Dynamik des Staates muß dabei die Legitimität und Progressivität seines Führungsanspruches hinterfragt werden. Die Staatsklasse verhält sich als ganze zwar bis zu einem gewissen Grad ökonomisch, in dem sie durchaus eine Kapitalakkumulation betreibt und so ihre materielle Basis sichert, sie ist aufgrund ihrer Monopolsituation aber keinem Konkurrenzdruck ausgesetzt. Ohne Konkurrenz, die für die kapitalistische Produktion konstitutiv ist, ist die Staatsklasse nicht zu ständiger Innovation gezwungen. Erhebliche Teile des abgeschöpften Mehrprodukts können verbraucht werden und müssen nicht produktiv eingesetzt werden. Ebenfalls wird der notwendige Interessenkonflikt zwischen Arbeit und Kapital, der in seiner Dialektik grundlegende Bedeutung für den Distributionsprozeß eines jeden kapitalistischen Systems besitzt, durch diese Staatsklasse unterdrückt. Aus dem Interesse nach einem möglichst hohen und wachsenden gesellschaftlichen Mehrprodukt heraus, ist einer Staatsklasse nichts an gleicher Verteilung der Einkommen gelegen, zumindest nicht im kurzfristigen Zeithorizont des sexenios, das den Rahmen für die Staatsklasse bestimmt. Die Untersuchung der internen Strukturen des mexikanischen Staates setzt zunächst an drei Machtfaktoren an: Die PRI ist zwar das politische Rückgrat des mexikanischen Staates, im Verlauf der "Institutionalisierung der Revolution" hat sie allerdings an Einfluß innerhalb des Staates verloren. Ihre Hauptaufgabe besteht in der Mobilisierung der Massen. Dem Präsidenten, mit einer auch formal äußerst weitgehenden Machtposition, die nur durch die Begrenzung der Amtszeit auf 6 Jahre eingeschränkt wird, steht eine wachsende Bürokratie gegenüber, die zunehmend selbst dazu übergeht als dominanter Faktor Politik zu machen. Diese Analyseform ist zwar hilfreich, übersieht aber, daß sich nicht hinter dieser Aufsplitterung der Machtfaktoren die eigentliche Dynamik des Staates verbirgt, da es sich dabei eher um eine zwar konfliktreiche aber funktionale Arbeitsteilung handelt. Vielmehr muß eine weitere Dimension eingeführt werden, die die Gesamtstruktur des Staates quer durchzieht. In einem Gesellschaftsystem, in dem der Staat eine ökonomische und politische Dominanz erlangt, besteht die Triebfeder staatlichen Handelns aus einem Widerspruch einerseits aus einem Legitimations- und Lei-

61 Uber die Eigentümlichkeit des mexikanischen Systems der Stabilität durch Integration: Adler Hellmann, J., Social Control in Mexico, in: Comparative Politics, (12-2), 1980, S. 226; Camacho, M., Control sobre el movimiento obrero en México, in: Fronteras ... (Fn. 59), S. 86; Grindle, M. S., Bureaucrats, Politicans and Peasants in Mexico, Los Angeles 1977, S. 182; Hansen, (Fn. 57), S. 222; Eckstein, S., Impact of Revolution, A. Comprehensive Analysis of Mexico and Bolivia, London 1976, S. 40; Rott, (Fn. 42); Kaufman Purcell, S., The Mexican Profit-Sharing Decision, Berkeley 1975; Stevens, (Fn. 58), S. 427; Mols, (Fn. 59), S. 401; Basáǹez, M., La lucha por la hegemonía en México 1968-1980, México 1981, S. 175.

62 Dazu vor allem Elsenhans, (Fn. 46), S. 118-190, der den Begriff der Staatsklasse einführte. 
stungsdruck auf den Staat von außen, d. h. von anderen privilegierten Gesellschaftsschichten. Andererseits sind die einzelnen Mitglieder an ihrer Selbstprivilegierung interessiert, die sie dank ihrer relativen Unkontrolliertheit viel weitgehender durchsetzen können als etwa in kapitalistischen Systemen.

Die Bürokratie als hauptsächlicher Träger staatlichen Handelns erhält durch diesen Widerspruch eine ambivalente Funktionsweise. Die persönlichen Interessen der Funktionäre in einer unkontrollierten Bürokratie drücken sich durch Klientelismus, Korruption und Rivalität aus. Dem stehen die Interessen des Gesamtapparates an der Erhaltung seiner Macht und somit der Erhaltung des bestehenden Systems gegenüber. Durch diese doppelte Anforderung funktioniert der bürokratische Apparat zwar bis zu einem gewissen Grad rational und kommt den Anforderungen des Weber'schen Bürokratiemodells hinlänglich nach, zu einem großen Teil ist er aber von personalistischen Strukturen durchzogen, die nicht bloße Funktionsausfälle bzw. Relikte traditionaler Herrschaftsformen darstellen, sondern die integraler Bestandteil einer sich privilegierenden Klasse sind. Die Rivalität innerhalb der Staatsklasse macht es für jedes Mitglied existenziell erforderlich, Allianzen einzugehen. Es bestehen Verbindungen, die sich bereits in der Universität gebildet haben und sich bis zu den camarillas, den Netzwerken innerhalb der Führungselite, fortsetzen. Andererseits bilden sich ganze equipos, in denen die Untergebenen dem Chef in seinen neuen Aufgabenbereich, wie im Falle der Pemex, folgen. In allen diesen Gruppen rangiert die persönliche Loyalität weit vor sachlichen Qualifikationen. ${ }^{63}$ Die Bedeutung klientelistischer Strukturen erhöht sich noch durch den Präsidentenwechsel alle 6 Jahre. Für jeden Präsidenten ist es eine Frage des politischen Úberlebens, die Mitglieder seiner Allianz auf den wichtigsten Posten zu plazieren. Da die Allianz bis weit nach unten pyramidisch aufgebaut ist, bringt jeder Präsidentenwechsel, obwohl innerhalb der gleichen Partei, einen Turnover von über 30000 Posten mit sich. Dadurch erhöht sich die Konkurrenz innerhalb des Staatsapparates. ${ }^{64}$

In einer breit angelegten Aktion zur moralischen Erneuerung - renovación moral versucht der neue Präsident, wie jede neue Regierung, die, selbst für lateinamerikanische Verhältnisse, sehr hohe Korruption zu bekämpfen. Für Mexiko ungewöhnlich, werden dabei selbst höhere Bürokraten verfolgt. In etwa 100 Korruptionsfällen außerhalb der Pemex wurden Anschuldigungen von ca. 3 mrd US \$ erhoben. ${ }^{65}$ Addiert mit den oben behandelten Pemex-Korruptionsbeschuldigungen ergibt sich die Summe von etwa 3,5 mrd US \$ zuzüglich des für 10 mrd US \$ verschwundenen Erdöls. Da selbst die Regierung schätzt, daß kaum mehr als $1 \%$ der Korruptionsfälle aufgedeckt werden,

63 Vgl. vor allem die Studie von Grindle, (Fn. 61), Weiter: Sloan, J. W., Bureaucracy and Public Policy in Latin America, in: Inter-American Economic Affairs, (34-4), 1981; Eckstein, (Fn. 59), S. 408; Stevens, E. P., Protest and Response in Mexico, Cambridge, Mass. 1974, S. 85.

64 Fagen, R. R./Tuohy, W. S., Aspects of the Mexican Political System, in: Studies in Comparative International Development, (3), 1972, S. 211.

65 Zusammengestellt aus mexikanischen Zeitungen zwischen März und August 1983. Die Fälle stammen aus der Zeit zwischen 1980 und 1982. Es handelt sich um Betrug, Bestechung oder unerklärliche Bereicherung. 
dürfte die gesamte Korruption einen Großteil der Auslandsverschuldung ausmachen. Die auf "ein unfaßliches Niveau gestiegene Korruption in Mexiko" ${ }^{66}$ ist jedoch keine Frage der Moral der Bürokraten, sondern der sich bietenden Möglichkeiten. Am Korruptionsgrad kann einerseits das Ausmaß der Dominanz und der Unkontrolliertheit der Staatsklasse abgelesen werden und andererseits die Höhe der abschöpfbaren Geldmengen, die im Ölboom erheblich gestiegen waren. Im Interesse der Erhaltung des Gesamtapparates ist die Antikorruptionskampagne ein deutliches Zeichen für die Mitglieder der Staatsklasse, daß die Korruption ökonomisch vertretbare Ausmaße überschritten hat und daß sie neu organisiert werden muß.

\section{Folgerungen}

Treffen auf eine Ókonomie, die durch verzerrte Preise und damit heterogene Strukturen gekennzeichnet ist, stark erhöhte Renteneinkommen durch Erdölexporte und äußere Verschuldung, weist der unflexible Produktionsapparat nur eine geringe Absorptionsfähigkeit auf. Erhöhte Nachfrage löst Inflationsprozesse aus, die Einkommensungleichheit verschärft sich weiter. Der Olsektor, der in einer Wachstumsstrategie eine Motorenfunktion übernehmen soll, wird vermehrt zu einer Enklave ohne positive Rückwirkungen auf andere Sektoren. Da die hohen Geldzuflüsse nur zu einem Teil produktiv umgesetzt werden können, erhöht sich die Spekulation und die Kapitalflucht. Wird eine solche Gesellschaft zudem von einer dominanten Staatsklasse beherrscht, so kann diese durch erhöhte Einnahmen auch ihren ökonomischen Einfluß ausbauen, wirkt aber durch ihre zweifelhafte Wachstumspolitik und ihre Selbstprivilegierungstendenzen doppelt retardierend.

Es bleibt zu fragen, ob eine derart gelagerte Gesellschaftsstruktur überhaupt eine kapitalistische Produktionsweise, wenn auch in ihrer unterentwickelten Form, darstellt. Es besteht zwar eine Bourgeoisie und eine Arbeiterklasse, ihr Interessenkonflikt erlangt aber nicht eine gesellschaftlich dominante Qualität. Diese wird vielmehr durch die Dynamik der Staatsklasse bestimmt. Ǒkonomisch gesehen sind zwar die Akteure eines Kapitalismus vorhanden, entscheidende Mechanismen bleiben aber ausgeschaltet. Einerseits fehlt zu großen Teilen ökonomische Konkurrenz, die Innovationsprozesse erforderlich macht, andererseits kann durch das Úberangebot unqualifizierter Arbeitskraft das Lohnniveau nicht angehoben werden. Damit bleibt die Produktion für einen Massenmarkt unrentabel. Das bloße Ausbeutungsmoment ist kein hinreichendes Definitionsmerkmal einer kapitalistischen Produktionsweise, sondern bildet eine Charakteristik auch anderer arbeitsteiliger Gesellschaftsformationen. 


\title{
ABSTRACTS
}

\author{
The Republic of South Africa's New Constitution - from "Westminster" to "Southmin- \\ ster"
}

By Klaus Frhr. von der Ropp

The long-time watcher of the Southern African crisis scenarios in 1984 will come to the conclusion that white South Africa has regained its traditional position of power and self-confidence which it had lost after the collapse of the Portuguese empire in Africa, the coming to power of Robert G. Mugabe in Zimbabwe and the 1976/77 rebellion of black South Africa's youth. Foremost among numerous other factors, the failure of the West's Namibia and South Africa policy in 1977-81 contributed to this resurgence of strength. At the time the five members of the Western Contact group asked for a system of majority rule to be introduced in the former mandate territory of South West Africa/ Namibia. Three members of the group, namely the U.S., West Germany and Canada, held the view that this solution, intended for Namibia, would also serve as an example for the Republic of South Africa. Thus Western powers created high expectations among black South Africans and provoked fear even among the most liberal whites. As a consequence the existing polarisation between the white African nation and black South Africa was exacerbated. To most observers inside and outside South Africa it was hardly conceivable that the many progressive speeches of those years would not be followed by any action.

Today's debate on South Africa's new constitutional dispensation has to be seen against the above-mentioned background. Pretoria no longer sees the necessity of genuine political change. So, after long and intensive debates, a system of sham-consociationalism is established for white, 'coloured a and Indian South Africans, whereas the existing system of separation is maintained for the country's black majority. The fear therefore persists that, to quote Gatsha Buthelezi, future constitutional developments in South Africa are going to be a product of bullets and power.

\section{Oil, Economic Crisis and the State in Mexico}

\section{By Herbert Baumgartner}

Despite favourable conditions the Mexican economy recently tumbled into its most serious crisis since the 1930ies. Between 1978 and 1981 the oil industry was the key for high growth rates. PEMEX, the national oil company, experienced dynamic expansion, multiplying sales. Nevertheless it maintained inefficient structures and corruption became rampant. 
Subsequent high inflation rates, the contraction of the economy since 1982 and the giant foreign debt can be explained both by short-term economic decisions, and long-term structural causes. Deficit spending, to which production could not respond, and an overvaluation of the peso were the main obstacles that led to a surprising breakdown.

Moreover, Mexico shows a highly unequal distribution of income, as well as widely divergent productivity. A mere industrial growth strategy could not bring a solution as it does not create enough jobs for unqualified labour that consequently could not improve its income. However, such improvements is seen as a condition for higher demand and subsequent expansion of mass production. As unqualified labour remained cheap, its productivity would not be increased. Unequal productivity with biased price systems leaves some sectors unattractive to capital investors. Since there are no equalizing mechanisms, the economy remains divided hamstrung in the long-run.

In the face of further destabilization the state intervened in the economy and, within 10 years, has doubled its expenditure as percentage of the national product. The state of ers an alliance to the national bourgeoisies. The bourgeoisie, however, does not dominate the state. The incorporation of social groups affords the state considerable economic and political autonomy. In the interest of keeping power, state action, however, is not necessarily progressive. On the contrary, severe corruption shows that the state tends to privilege itself. Personalistic and clientelistic relations within a strong presidential system are ineffectual in handling economic problems.

\section{The Army Come Apart. On the Rôle of the Iranian Armed Forces During the Revolution 1978/79}

\section{By Rudolf Hamann}

The building of the Iranian army was the Shah's own work and had a two-fold purpose: protecting the state from attacks by other countries, like Irak or Russia, and securing the Shah's authority against his own people. After 1973, when the price of oil rose rapidly, the Shah became the best buyer of weapons in the United States of America. Five years later half a million men were under arms. To win a civil war, however, the soldiers were wrongly armed, and for a regular war the army was too weak because of an ineffective command chosen by nepotism instead of achievement. In addition to this only few officers and non-commissioned officers were able to motivate the soldiers. In 1978 the army therefore looked more like a large ordnance depot, completely disintegrated, unable to manage complex weapons and depending on American specialists.

The first riots in the beginning of 1978 were still bloodily suppressed by the army but in the autumn the generals were certain that the Shah régime could no longer be maintained by violence. When the Shah had left the country, the generals considered a putsch for a moment, but the U.S. refused support because of the army's isolation inside Iran. So the army disbanded by stages, and particularly the common soldiers deserted to Khomeini. 